\title{
Gain-Loss Separability and Coalescing in Risky Decision Making
}

\author{
Michael H. Birnbaum \\ Decision Research Center, Department of Psychology, California State University, \\ 800 North State College Boulevard, Fullerton, California 92834-6846, mbirnbaum@fullerton.edu \\ Jeffrey P. Bahra \\ California State University, Fullerton,
}

\begin{abstract}
$\mathrm{T}$ his experiment tested two behavioral properties of risky decision making-gain-loss separability (GLS) and coalescing. Cumulative prospect theory (CPT) implies both properties, but the transfer of attention exchange model (TAX) violates both. Original prospect theory satisfies GLS but may or may not satisfy coalescing, depending on whether editing rules are assumed. A configural form of CPT proposed by Wu and Markle (2004) violates GLS, but satisfies coalescing. New tests were designed and conducted to test these theories against specific predictions of a TAX model. This model used parameters estimated from previous data, together with simple new assumptions to extend TAX to gambles with negative and mixed consequences. Contrary to all three forms of prospect theory, systematic violations of both coalescing and of GLS were observed. Violations of GLS were confirmed by analyses of individual data patterns by means of an error model in which each choice can have a different rate of error. Without estimating any parameters from the new data, the TAX model predicted the majority choices in the new data fairly well, correctly predicting when modal choices would violate GLS, when they would satisfy it, and when indifference would be observed.
\end{abstract}

Key words: utility preference; decision choice; decision risk

History: Accepted by Detlof von Winterfeldt, decision analysis. This paper was with the authors 1 month and

3 weeks for 1 revision.

\section{Introduction}

This study is based upon an important paper by Wu and Markle (2004). It not only replicates and extends their results, it also provides new tests of the model they proposed to account for their data. In addition, this paper provides a different theoretical interpretation of their findings, and it tests that alternative model. Wu and Markle (2004) reported systematic violations of a behavioral property known as gain-loss separability (GLS), which is implied by many descriptive decision models.

\subsection{Gain-Loss Separability (GLS)}

GLS is a behavioral property defined on choices between mixed gambles (gambles containing both gains and losses). Let $G=\left(y_{1}, p_{1} ; y_{2}, p_{2} ; \ldots ; y_{n}, p_{n}\right.$; $\left.x_{m}, q_{m} ; \ldots ; x_{2}, q_{2} ; x_{1}, q_{1}\right)$ represent a mixed gamble in which the outcomes are ranked such that $y_{1}<$ $y_{2}<\cdots<y_{n}<0<x_{m}<\cdots<x_{2}<x_{1}$ and $\sum_{i=1}^{n} p_{i}+$ $\sum_{j=1}^{m} q_{j}=1$. Now break $G$ into positive and negative subgambles, as follows: $G^{+}=\left(0, \sum_{i=1}^{n} p_{i} ; x_{m}\right.$, $\left.q_{m} ; \ldots ; x_{2}, q_{2} ; x_{1}, q_{1}\right)$ and $G^{-}=\left(y_{1}, p_{1} ; y_{2}, p_{2} ; \ldots ; y_{n}\right.$, $\left.p_{n} ; 0, \sum_{j=1}^{m} q_{j}\right)$. Let $F$ represent another mixed gamble that can also be broken into positive and negative subgambles.
GLS is the assumption that if a person prefers the positive subgamble $G^{+}$to the positive subgamble $F^{+}$ and prefers the negative subgamble $G^{-}$to the negative subgamble $F^{-}$, then that person should prefer $G$ to $F$. That is, with $\succ$ denoting preference, if $G^{+} \succ F^{+}$ and $G^{-} \succ F^{-}$, then $G \succ F$. As shown in the next section, GLS is implied by any theory in which the utility of $G$ can be written as a strictly increasing function of the utility of $G^{+}$and the utility of $G^{-}$. The violations of GLS observed by Wu and Markle (2004) therefore refute this class of theories, which includes cumulative prospect theory $(\mathrm{CPT})$.

\subsection{Cumulative Prospect Theory (CPT)}

Let $G=\left(y_{1}, p_{1} ; y_{2}, p_{2} ; \ldots ; y_{n}, p_{n} ; x_{m}, q_{m} ; \ldots ; x_{2}, q_{2} ; x_{1}\right.$, $q_{1}$ ) represent a mixed gamble with outcomes ranked such that $y_{1}<y_{2}<\cdots<y_{n}<0 \leq x_{m}<\cdots<x_{2}<$ $x_{1}$. Define cumulative probabilities of losses as $P_{i}=$ $\sum_{k=1}^{i} p_{k}$, and define decumulative probabilities of gains as $Q_{j}=\sum_{k=1}^{j} q_{k}$. CPT (Tversky and Kahneman 1992) can be written as follows:

$$
\begin{aligned}
\operatorname{CPU}(G)= & \sum_{i=1}^{n}\left[W^{-}\left(P_{i}\right)-W^{-}\left(P_{i-1}\right)\right] u\left(y_{i}\right) \\
& +\sum_{j=1}^{m}\left[W^{+}\left(Q_{j}\right)-W^{+}\left(Q_{j-1}\right)\right] u\left(x_{j}\right),
\end{aligned}
$$


where $P_{i}$ and $P_{i-1}$ are the probabilities of a loss being equal to or worse (lower) than $y_{i}$ and strictly lower than $y_{i}$, respectively; $Q_{j}$ and $Q_{j-1}$ are the probabilities of winning a positive prize of $x_{j}$ or more, and strictly more than $x_{j}$, respectively $\left(P_{0}=Q_{0}=0\right)$. Utility is defined with respect to changes from the status quo (gains or loses), where $u(0)=0 . C P U(G)$ is the utility ("subjective value") of the gamble; the representation assumes that $G \succ F \Leftrightarrow C P U(G) \succ C P U(F)$. The functions $W^{+}(Q)$ and $W^{-}(P)$ are strictly increasing probability-weighting functions, $W^{+}(0)=W^{-}(0)=0$, and $W^{+}(1)=W^{-}(1)=1$.

From Equation (1), it can be seen that the overall utility of a mixed gamble in CPT is just the sum of the utilities of its positive and negative subgambles. This additivity of the favorable (positive) and unfavorable (negative) parts of a gamble implies GLS. Equation (1) implies $C P U(G)=C P U\left(G^{+}\right)+C P U\left(G^{-}\right)$for all mixed gambles, $G$. CPT assumes that $G^{+} \succ F^{+} \Leftrightarrow$ $\operatorname{CPU}\left(G^{+}\right)>\operatorname{CPU}\left(F^{+}\right)$and $G^{-} \succ F^{-} \Leftrightarrow \operatorname{CPU}\left(G^{-}\right)>$ $\operatorname{GPU}\left(F^{-}\right)$. If both conditions hold, then it follows that $C P U\left(G^{+}\right)+C P U\left(G^{-}\right)>C P U\left(F^{+}\right)+C P U\left(F^{-}\right) \Leftrightarrow G \succ F$. The same implication follows for any such additive theory, including original prospect theory. GLS is also implied by rank- and sign-dependent utility theory (Luce and Fishburn 1991, 1995), which used the same additive representation later used in CPT (see Luce 2000, Chapters 6 and 7 for contrasting approaches).

The weighting functions in CPT have been further specified as follows:

$$
\begin{gathered}
W^{+}(Q)=\frac{Q^{\gamma}}{\left[Q^{\gamma}+(1-Q)^{\gamma}\right]^{1 / \gamma}} \text { and } \\
W^{-}(P)=\frac{P^{\delta}}{\left[P^{\delta}+(1-P)^{\delta}\right]^{1 / \delta}},
\end{gathered}
$$

where the constants $\gamma$ and $\delta$ were estimated by Tversky and Kahneman (1992) to be 0.61 and 0.69, respectively; $u(x)$ was approximated by $u(x)=x^{\beta}$, where $\beta=0.88, x>0$, and $u(-x)=-\lambda u(x) ; x \geq 0$. The constant $\lambda$ is sometimes referred to as the "loss aversion" coefficient; $\lambda$ was estimated to be 2.25 . This paper will evaluate both the general model (Equation (1)), which implies GLS, as well as the above parameterized version of Tversky and Kahneman (1992) in order to relate new findings to previous results and to show where the CPT model goes wrong.

\subsection{Violations of GLS}

$\mathrm{Wu}$ and Markle (2004) reported systematic violations of GLS, illustrated by the three choices in Table 1. A majority of respondents preferred $F$ to $G$ in Choice 3, replicating a pattern found by Levy and Levy (2002), which is consistent with CPT as fit by Tversky and Kahneman (1992) to previous data (Wakker 2003). To test GLS, Wu and Markle (2004) decomposed G and F into their gain and loss subgambles. These choices are shown with their respective choice percentages. Contrary to GLS, the majority $(72 \%)$ preferred $\mathrm{G}^{+}$over $\mathrm{F}^{+}$ in Choice 1; 60\% preferred $G^{-}$over $F^{-}$in Choice 2; but $62 \%$ chose $F$ over $G$ in Choice 3 (Wu and Markle 2004).

Wu and Markle (2004) proposed a configural model of CPT to account for violations of GLS by allowing different weighting functions for the case of mixed gambles from those used for purely positive or purely negative gambles. Their model will be referred to as configural cumulative prospect theory (CCPT) because it retains the equations of CPT but uses different weighting functions for different configurations of consequences: positive, negative, and mixed:

$$
\operatorname{CCPU}\left(G^{+}\right)=\sum_{j=1}^{m}\left[W^{+}\left(Q_{j}\right)-W^{+}\left(Q_{j-1}\right)\right] u\left(x_{j}\right),
$$

\begin{tabular}{|c|c|c|c|c|c|}
\hline No. & $F^{+}, F^{-}$, or $F$ & $G^{+}, G^{-}$, or $G$ & $\% G$ & TAX & СРT \\
\hline 1 & 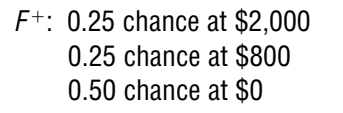 & $\begin{array}{c}G^{+}: 0.25 \text { chance at } \$ 1,600 \\
0.25 \text { chance at } \$ 1,200 \\
0.50 \text { chance at } \$ 0\end{array}$ & 72 & $497<552$ & $601>551$ \\
\hline 2 & $\begin{aligned} F^{-}: & 0.50 \text { chance at } \$ 0 \\
& 0.25 \text { chance at }-\$ 800 \\
& 0.25 \text { chance at }-\$ 1,000\end{aligned}$ & $\begin{aligned} G^{-}: & 0.50 \text { chance at } \$ 0 \\
& 0.25 \text { chance at }-\$ 200 \\
& 0.25 \text { chance at }-\$ 1,600\end{aligned}$ & 60 & $-359<-276$ & $-379>-437$ \\
\hline 3 & $\begin{array}{c}F: \quad 0.25 \text { chance at } \$ 2,000 \\
0.25 \text { chance at } \$ 800 \\
0.25 \text { chance at }-\$ 800 \\
0.25 \text { chance at }-\$ 1,000\end{array}$ & 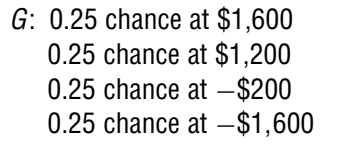 & 38 & $-280>-300$ & $-107.2>-178.6$ \\
\hline
\end{tabular}

Table 1 A Test of Gain-Loss Separability from Wu and Markle (2004)

Notes. Calculations show that the violation observed by Wu and Markle (2004) is consistent with the special TAX model. The column labeled TAX shows the calculated certainty equivalents for strictly nonnegative gambles and mixed gambles using Equation (4) with $u(x)=x, t(p)=p^{0.7}$, and $\delta=1$. The certainty equivalents of strictly nonpositive gambles are calculated by substituting absolute values in Equation (4) and multiplying the result by -1 . The column labeled CPT shows certainty equivalents from Equations (2) and (3) with $\gamma=0.61, \delta=0.69, u(x)=x^{\beta}, x>0$, where $\beta=0.88$; and $u(-x)=-\lambda u(x), x \geq 0$, where $\lambda=2.25$. 


$$
\begin{gathered}
\operatorname{CCPU}\left(G^{-}\right)=\sum_{i=1}^{n}\left[W^{-}\left(P_{i}\right)-W^{-}\left(P_{i-1}\right)\right] u\left(x_{i}\right), \\
\operatorname{CCPU}(G)=\sum_{i=1}^{n}\left[W^{-+}\left(P_{i}\right)-W^{-+}\left(P_{i-1}\right)\right] u\left(x_{i}\right) \\
+\sum_{j=1}^{m}\left[W^{+-}\left(Q_{j}\right)-W^{+-}\left(Q_{j-1}\right)\right] u\left(x_{j}\right),
\end{gathered}
$$

where the terms are as defined in Equation (1), except that $W^{-}$and $W^{+}$are the weighting functions for gambles composed of purely nonpositive consequences and purely nonnegative consequences, respectively, and the functions $W^{-+}$and $W^{+-}$are the weighting functions for the negative and positive components of mixed gambles, respectively. This approach recalls a suggestion by Edwards (1962), who proposed that different weighting functions may be required for gambles with strictly positive, strictly nonnegative (including zero), strictly negative, strictly nonpositive (including zero), and mixed consequences. Although CCPT violates GLS, it must satisfy the property of coalescing (described next) that is implied by these equations.

\subsection{Coalescing}

Coalescing is the assumption that if there are two probability-consequence branches in a gamble leading to the same consequence, they can be combined by adding their probabilities. For example, consider gamble $A=(\$ 100,0.25 ; \$ 100,0.25 ; \$ 0,0.5)$. Gamble $A$ is a three-branch gamble in which one branch has a probability of 0.5 to win $\$ 0$, and two other branches of probability 0.25 to win $\$ 100$.

According to the property of coalescing, this threebranch gamble, $A$, is equivalent to the two-branch gamble, $A^{\prime}=(\$ 100,0.5 ; \$ 0,0.5)$. Gamble $A^{\prime}$ is called the coalesced form of the gamble, and $A$ is one of many possible split forms. Assuming transitivity, coalescing implies that people should make the same choice between $A^{\prime}$ and $B$ as they do between $A$ and $B$, apart from random error.

In original prospect theory (Kahneman and Tversky 1979), coalescing was assumed as an editing rule (combination), but is not implied by the equations. Therefore, depending on whether we accept the editing rule or equations, original prospect theory either satisfies or violates coalescing.

In CPT or CCPT, however, coalescing follows from the rank-dependent representation (Birnbaum and Navarrete 1998, pp. 57-58; Luce 2000). A number of studies, however, reported systematic evidence against coalescing (Starmer and Sugden 1993; Humphrey 1995; Birnbaum 2004a, b). Such violations are consistent with the older class of configural weight models (Birnbaum and Stegner 1979) that preceded the class that have come to be known as rank dependent, including CPT.

\subsection{Transfer of Attention Exchange Model (TAX)}

The TAX model is a type of the configurally weighted averaging model in which the weights of branches are affected by the ranks of the consequences on those branches. Such models were developed to account for integrated psychophysical and evaluative judgments in psychology (Birnbaum 1974, Birnbaum and Stegner 1979). Although they have some similarity to models that were later introduced as rank-dependent utility models (Quiggin 1982, 1993), including CPT, they differ in important respects. These models violate coalescing and attribute the Allais paradoxes to violations of coalescing (Birnbaum 1999a, 2004a) rather than to violations of "independence," as is done in CPT.

The basic ideas of the TAX model are as follows: A risky gamble is represented as a tree with probability-consequence branches. Aside from configural effects, the weight of each branch is a function of the branch's probability. However, weight is transferred from branch to branch according to the ranks of the consequences on those branches. Intuitively, these transfers of weight represent shifts of attention among the branches. In a risk-averse person, branches leading to lower consequences end up with more weight. Luce and Marley (2005) have axiomatized a general form of TAX and have shown that it is an idempotent, rank-weighted utility model. In the so-called "special" TAX model (Birnbaum and Chavez 1997), a simpler form of TAX, all transfers of weight between any two branches represent the same proportion of the probability weight of the branch giving up its weight.

Consider gambles of positive consequences, $G=$ $\left(x_{1}, p_{1} ; x_{2}, p_{2} ; \ldots ; x_{n}, p_{n}\right)$, with $n$ distinct branches, where the consequences are ordered such that $x_{1}>$ $x_{2}>\cdots>x_{n} \geq 0$. The special TAX model (for the case where $\delta \geq 0$ ) can be written as follows:

$$
\begin{array}{r}
\operatorname{TAX}(G)=\left(\sum _ { i = 1 } ^ { n } u ( x _ { i } ) \left[t\left(p_{i}\right)-\frac{\delta}{(n+1)} \sum_{k=i+1}^{n} t\left(p_{i}\right)\right.\right. \\
\left.\left.+\frac{\delta}{(n+1)} \sum_{k=1}^{i-1} t\left(p_{k}\right)\right]\right) \cdot\left(\sum_{i=1}^{n} t\left(p_{i}\right)\right)^{-1},
\end{array}
$$

where the configural transfer parameter is $\delta$. When $\delta=0$ and $t(p)=p$, this model reduces to expected utility (EU). The effect of $\delta$ is to transfer weight from one branch to another, representing attention redirected from branch to branch. In this model, risk aversion is produced when weight is taken from branches with higher-valued consequences and given to branches with lower-valued consequences. This aspect of the model can be contrasted with the attribution of risk aversion to the shape of the utility function (as in EU).

To further understand the model, consider the three terms in the numerator representing the weight of (attention given to) the branch leading to consequence $x_{i}$. The first term, $t\left(p_{i}\right)$, represents the weight 
as a function of the branch's probability; if $\delta=0$, the relative weight would simply be the ratio of $t\left(p_{i}\right)$ to the sum of these transformed probabilities. The second term represents weight given up by this branch to branches with lower consequences. Note that each term in this sum is the same; i.e., all are proportional to $t\left(p_{i}\right)$ and there are $n-i$ of them, so this term reduces to $-\delta(n-i) t\left(p_{i}\right) /(n+1)$. The third term represents attention transferred from other branches (with higher consequences) to this branch. Each of these terms is proportional to the transformed probabilities of the branches giving up weight. However, the sum of weight transfers is zero; that is, weight is neither created nor destroyed, but only transferred from branch to branch.

In this paper, we use the parameters of Birnbaum (1999a), which were chosen to approximate the data of Tversky and Kahneman (1992) for certainty equivalents of gambles of the form $(\$ 100, p ; \$ 0)$. These parameters are $t(p)=p^{0.7}, \delta=1$, and $u(x)=x$ for $0 \leq$ $x \leq \$ 100$. (Equation (4) is written here with branches ordered from best to worst; therefore, the value of $\delta=1$ here corresponds to $\delta=-1$ in Birnbaum and Chavez 1997 and Birnbaum 1999a, b, which used different conventions.) Birnbaum (1999a) showed that these same parameters also give a good fit to other data involving gambles on gains, including other results that contradict $\mathrm{CPT}$.

The special TAX model has been defined for gambles with strictly nonnegative consequences. To apply the model to gambles with purely negative consequences and mixed gambles, it is necessary to make some additional assumptions.

\subsection{TAX Extended to Gambles with Negative and Mixed Consequences}

First, assume that $u(x)=x$ for $-\$ 100 \leq x \leq \$ 100$. This assumption is quite different from what is done in $\mathrm{CPT}$, where it is assumed that there is a kink in the utility function at zero.

Second, gambles with strictly nonpositive consequences are calculated by substituting absolute values for the consequences in Equation (4) (using the same parameters) and multiplying the resulting positive utility by -1 . This assumption implies the "reflection" property: If gambles with strictly nonnegative consequences are converted to strictly nonpositive ones by multiplying each consequence by -1 , then the order of preferences of the reflected gambles is reversed relative to that of the original gambles. For example, if $A=(x, p ; y, 1-p) \succ B=\left(x^{\prime}, p^{\prime} ; y^{\prime}, 1-\right.$ $\left.p^{\prime}\right)$, where all the consequences are nonnegative, then $-A=(-x, p ;-y, 1-p) \prec-B=\left(-x^{\prime}, p^{\prime} ;-y^{\prime}, 1-p^{\prime}\right)$. This property has been found to be descriptive in a number of studies, although not all (see the review in Starmer 2000). This study will test this prediction, which is implied by both CPT and this TAX model.
Third, mixed gambles, $G=\left(x_{1}, p_{1} ; x_{2}, p_{2} ; \ldots ; x_{m}, p_{m} ;\right.$ $\left.x_{m+1}, p_{m+1} ; x_{m+2}, p_{m+2} ; \ldots ; x_{n}, p_{n}\right)$, where $x_{1}>x_{2}>\cdots>$ $x_{m} \geq 0>x_{m+1}>x_{m+2}>\cdots>x_{n}$, as well as gambles composed of strictly nonnegative consequences [ $G=$ $\left.\left(x_{1}, p_{1} ; x_{2}, p_{2} ; \ldots ; x_{n}, p_{n}\right)\right]$, where $x_{1}>x_{2}>\cdots>x_{n} \geq 0$, are both calculated by substituting algebraic values into expression (4). Note that the ranking here is also based on algebraic value. This assumption represents both risk aversion (tendency to prefer a sure gain over a gamble with the same expected value (EV)) and loss aversion (tendency to prefer a sure gain over a mixed gamble with the same EV) by the same weighting mechanism; it assumes that both phenomena are governed by the same parameter, $\delta$. This study tests risk aversion and loss aversion, which are implied by both CPT and TAX.

With these three assumptions, no new parameters are needed to apply the special TAX model to gambles with negative and mixed consequences. These assumptions and parameters are listed in Table 1 for easy reference throughout the rest of this paper. This model has fewer parameters than CPT because it does not use a loss aversion parameter and it does not have different weighting functions for positive and negative consequences. It is also more parsimonious than CCPT, which uses a third probability-weighting function for mixed gambles.

\subsection{TAX Violates GLS}

Table 1 displays calculated certainty equivalents (CE) of gambles in the $\mathrm{Wu}$ and Markle (2004) test, where $\mathrm{CE}$ is the amount of cash that is theoretically indifferent to the gamble. These have been calculated for both CPT and TAX using the parameters in Table 1.

Although violations of GLS (Table 1) refute CPT with any parameters (CPT implies GLS), the violation in Table 1 is consistent with the special TAX model. The calculated utilities under the TAX model are shown in Table 1, where $\operatorname{TAX}\left(G^{+}\right)>\operatorname{TAX}\left(F^{+}\right)$, $\operatorname{TAX}\left(G^{-}\right)>\operatorname{TAX}\left(F^{-}\right)$and $\operatorname{TAX}(G)<\operatorname{TAX}(F)$, respectively, correctly reproducing modal choices observed by $\mathrm{Wu}$ and Markle that violate GLS.

Although no version of Equation (1) can explain systematic violations of GLS, it is interesting to examine where the parameterized model of CPT (which includes Equations (2)) fails. It is perhaps ironic that it is not in the choice between $G$ and $F$ where the parameterized versions of TAX and CPT disagree (and where Levy and Levy 2002 and Wakker 2003 disputed CPT). Rather, it is in the two choices between the three-branch subgambles where TAX and CPT disagree and where the CPT model with parameters of Tversky and Kahneman (1992) makes erroneous predictions (Table 1).

Note that the test of GLS in Table 1 involves three choices; two of them are between three-branch gambles and one is between four-branch gambles. This 
shift in the number of branches suggests that coalescing may play a role in this test. Examining the definition of the subgambles, we see that consequences below zero are set to zero and added to define $G^{+}$, and consequences above zero are set to zero and their probabilities are added to define $G^{-}$. The use of addition to combine the probabilities shows that coalescing has been assumed in the definitions of the subgambles.

\subsection{Purposes of the Present Study}

To investigate possible connections with coalescing, to test specific predictions of the TAX model, and to test the CCPT model of $\mathrm{Wu}$ and Markle (2004), we first simplified the example of Table 1, and then extended it. Note that Choices 4, 5, and 6 in Table 2 can be compared to Choices 1, 2, and 3 of Table 1, respectively. The revised cash consequences are equally spaced, but the new choices preserve the relative spreads of consequences used in the $\mathrm{Wu}$ and Markle (2004) example. In addition, Choices 6 and 7 provide a test of coalescing, and Choices 4, 5, and 7 constitute a new test of GLS with all three-branch gambles.

This design allows tests of GLS with all gambles having three branches (Choices 4, 5, and 7), or with three, three, and four branches (Choices 4, 5, and 6). In addition, the symmetric design with equally-spaced consequences permits a specific test of indifference between $G$ and $F$ in the split form (Choice 6), implied by TAX with the assumptions of Table 1 .

Qualitative properties of the models are summarized in Table 3. Table 3 shows implications of general forms of the models: CPT implies GLS and coalescing. CCPT violates GLS but satisfies coalescing. Original prospect theory (OPT) satisfies GLS and may or may not satisfy coalescing (depending on editing). TAX violates both properties. Table 2 summarizes specific

Table 2 New Tests, Designed by the TAX Model to Predict Violations of Gain-Loss Separability

\begin{tabular}{|c|c|c|c|c|}
\hline No. & $F^{+}, F^{-}$, or $F$ & $G^{+}, G^{-}$, or $G$ & & \\
\hline 4 & 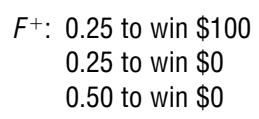 & $\begin{aligned} G^{+}: & 0.25 \text { to win } \$ 50 \\
& 0.25 \text { to win } \$ 50 \\
& 0.50 \text { to win } \$ 0\end{aligned}$ & 13.8 & 20.6 \\
\hline 5 & $\begin{aligned} F^{-}: & 0.50 \text { to lose } \$ 0 \\
& 0.25 \text { to lose } \$ 50 \\
& 0.25 \text { to lose } \$ 50\end{aligned}$ & $\begin{aligned} G^{-}: & 0.50 \text { to lose } \$ 0 \\
& 0.25 \text { to lose } \$ 0 \\
& 0.25 \text { to lose } \$ 100\end{aligned}$ & -20.6 & -13.8 \\
\hline 6 & $\begin{aligned} & F: 0.25 \text { to win } \$ 100 \\
& 0.25 \text { to win } \$ 0 \\
& 0.25 \text { to lose } \$ 50 \\
& 0.25 \text { to lose } \$ 50\end{aligned}$ & $\begin{array}{l}\text { G: } 0.25 \text { to win } \$ 50 \\
0.25 \text { to win } \$ 50 \\
0.25 \text { to lose } \$ 0 \\
0.25 \text { to lose } \$ 100\end{array}$ & -25.0 & -25.0 \\
\hline 7 & 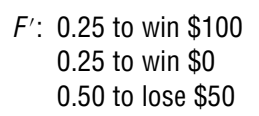 & $\begin{aligned} G^{\prime}: & 0.50 \text { to win } \$ 50 \\
& 0.25 \text { to lose } \$ 0 \\
& 0.25 \text { to lose } \$ 100\end{aligned}$ & -15.5 & -34.5 \\
\hline
\end{tabular}

Note. Choices 6 and 7 represent the same choice, except for coalescing. TAX implies indifference in Choice 6.

\begin{tabular}{l} 
Table $3 \begin{array}{c}\text { Relationship Among Models with Respect } \\
\text { to the Behavioral Properties of Gain-Loss } \\
\text { Separability (GLS) and Coalescing }\end{array}$ \\
\hline GLS Coalescing \\
Satisfied \\
Violated \\
Notes. OPT = original prospect theory (Kahneman and \\
Tversky 1979); OPT either does or does not satisfy coa- \\
lescing, depending on whether one assumes the edit- \\
ing rule of combination or not, respectively. CPT = \\
cumulative prospect theory (Tversky and Kahneman \\
1992). CCPT is the modified version of CPT presented \\
by Wu and Markle (2004), in which the cases of strictly \\
positive, negative, and mixed gambles are handled with \\
the rank-dependent representation with different weight- \\
ing functions in each of these three cases. TAX refers \\
to the special case of Birnbaum's TAX model, in which \\
the same configural weight parameter applies to strictly \\
nonnegative and mixed gambles, and strictly nonpositive \\
gambles are calculated by reflection.
\end{tabular}

numerical predictions of TAX combined with its previous parameters and assumptions (Table 1). Choices 4,5 , and 7 should show violation of GLS. However, Choices 6 and 7 should yield quite different results (from each other) because TAX implies a violation of coalescing, and Choice 6 should be consistent with a choice probability of 0.5 because the two gambles have equal utilities according to TAX with the assumptions in Table 1. This study also uses additional tests of GLS and coalescing, including cases where GLS should be violated (Choices 4, 5, and 7, all with three branches) as well as cases where it should be satisfied (shown later) according to the TAX model.

These properties will be tested not only at the level of modal choices, as in Table 1, but also with respect to individual choice patterns. A true and error model that allows individual differences in "true" preference patterns will be used to separate true preferences from those produced by "error" in the data.

\section{Method}

Participants completed this study as two parts in a series of 12 decision tasks, each consisting of 20-30 choices. This experiment consisted of 21 choices that were presented twice, separated by five other tasks requiring an intervening time of about 25 minutes. Participants viewed the materials via the World Wide Web. Working at their own paces, most completed the series of 12 tasks (including both parts of this study) in one and one-half to two hours. Each choice was displayed as in the following example:

1. Which do you choose?

$\square$ A: 50 black marbles to win $\$ 100$ 50 red marbles to lose $\$ 100$ 
OR

$$
\text { B: } 50 \text { green marbles to win } \$ 1
$$$$
50 \text { yellow marbles to lose } \$ 1
$$

Each gamble was described in terms of a container holding exactly 100 marbles of different colors, from which one marble would be drawn at random, and the color of that marble would determine the prize. In the choice illustrated above, Gamble $A$ is a $50-50$ chance to win $\$ 100$ or to lose $\$ 100$, and Gamble $B$ represents a 50-50 chance to win $\$ 1$ or lose $\$ 1$.

Participants were instructed to click a button beside the gamble that they would prefer to play in each choice. They were informed that three lucky participants per 100 would receive the prize of one of their chosen gambles. They were informed that these would receive $\$ 100$ plus the consequence of one of their chosen gambles. They could thus win up to $\$ 200$ or end up with as little as $\$ 0$. (Prizes were awarded as promised to six people.) According to Kahneman and Tversky (1979), such an endowment should have no effect, an assumption they used to account for "framing" effects. Possible effects of the endowment of $\$ 100$ will be considered later.

\subsection{Experimental Design}

Tables 4 and 5 show choices used to test GLS and coalescing. Table 6 shows "filler" choices that assessed risk attitude, loss attitude, and idempotence (the assumption that if all consequences are the same, the gamble is equivalent to the sure thing of that conse-
Complete instructions and materials are available on the Management Science website at http://mansci. pubs.informs.org/ecompanion.html.

\subsection{Participants}

Participants were 178 undergraduates enrolled in lower-division psychology at California State University, Fullerton. About half were tested on Internetconnected computers in labs, and the rest completed the studies at times and places of their own choosing via the World Wide Web. There were 137 females and 41 males; $71 \%$ were $\leq 19$ years of age, and $93 \%$ were $\leq 22$ years.

\section{Results}

Table 4 shows the percentage choosing the second $(G)$ gamble in each choice of the main design. Choices 15, 13, and 11 form a test of GLS with three-branch gambles. The table shows that $71 \%$ chose $\mathrm{G}^{+}$over $\mathrm{F}^{+}$and $65 \%$ chose $G^{-}$over $F^{-}$; however, $76 \%$ chose $F$ over $G$ in Choice 11, violating GLS. All three percentages are significantly different from $50 \%$, but in opposite directions $(z=6.48,4.60$, and -7.83 , respectively). These modal choices are consistent with the predictions of TAX (with parameters in Table 1), but they violate CPT with any functions for $W(P)$ and $u(x)$.

Choice 19 in Table 4 is the same as Choice 11, except for splitting/coalescing. The TAX model correctly predicted that the majority would choose $F$ in Choice 11 (76\% did so), and it predicted indifference in Choice 19, where the empirical choice proportion is 0.517 . The small discrepancy between 0.517 and 0.500

Table 4 Tests of Gain-Loss Separability and Coalescing

\begin{tabular}{|c|c|c|c|c|c|c|c|}
\hline \multirow[b]{2}{*}{ No. } & \multicolumn{2}{|c|}{ Choice } & \multirow[b]{2}{*}{$\% G$} & \multicolumn{2}{|c|}{ Prior TAX } & \multicolumn{2}{|c|}{ Prior CPT } \\
\hline & First gamble, $F$ & Second gamble, $G$ & & $F$ & G & $F$ & G \\
\hline 15 & $\begin{array}{l}25 \text { black to win } \$ 100 \\
25 \text { white to win } \$ 0 \\
50 \text { white to win } \$ 0\end{array}$ & $\begin{array}{l}25 \text { blue to win } \$ 50 \\
25 \text { blue to win } \$ 50 \\
50 \text { white to win } \$ 0\end{array}$ & 0.71 & 13.8 & 20.6 & 24.6 & 18.7 \\
\hline 13 & $\begin{array}{l}50 \text { white to lose } \$ 0 \\
25 \text { pink to lose } \$ 50 \\
25 \text { pink to lose } \$ 50\end{array}$ & $\begin{array}{l}50 \text { white to lose } \$ 0 \\
25 \text { white to lose } \$ 0 \\
25 \text { red to lose } \$ 100\end{array}$ & 0.65 & -20.6 & -13.8 & -20.4 & -24.8 \\
\hline 19 & $\begin{array}{l}25 \text { black to win } \$ 100 \\
25 \text { white to win } \$ 0 \\
25 \text { pink to lose } \$ 50 \\
25 \text { pink to lose } \$ 50\end{array}$ & $\begin{array}{l}25 \text { blue to win } \$ 50 \\
25 \text { blue to win } \$ 50 \\
25 \text { white to lose } \$ 0 \\
25 \text { red to lose } \$ 100\end{array}$ & 0.52 & -25.0 & -25.0 & -8.8 & -15.3 \\
\hline 11 & $\begin{array}{l}25 \text { black to win } \$ 100 \\
25 \text { white to win } \$ 0 \\
50 \text { pink to lose } \$ 50\end{array}$ & $\begin{array}{l}50 \text { blue to win } \$ 50 \\
25 \text { white to lose } \$ 0 \\
25 \text { red to lose } \$ 100\end{array}$ & 0.24 & -15.5 & -34.5 & -8.8 & -15.3 \\
\hline 17 & $\begin{array}{l}25 \text { black to win } \$ 100 \\
25 \text { white to win } \$ 0 \\
25 \text { white to lose } \$ 0 \\
25 \text { red to lose } \$ 100\end{array}$ & $\begin{array}{l}25 \text { blue to win } \$ 50 \\
25 \text { blue to win } \$ 50 \\
25 \text { pink to lose } \$ 50 \\
25 \text { pink to lose } \$ 50\end{array}$ & 0.57 & -30.0 & -20.0 & -12.8 & -11.2 \\
\hline
\end{tabular}

Notes. Prior TAX and Prior CPT refer to the models plus the previously estimated parameters and assumptions listed in the notes to Table 1. 


\begin{tabular}{|c|c|c|c|c|c|c|c|}
\hline \multirow[b]{2}{*}{ No. } & \multicolumn{2}{|c|}{ Choice } & \multirow[b]{2}{*}{$\% G$} & \multicolumn{2}{|c|}{ Prior TAX } & \multicolumn{2}{|c|}{ Prior CPT } \\
\hline & First gamble, $F$ & Second gamble, $G$ & & $F$ & G & $F$ & G \\
\hline 15 & $\begin{array}{l}25 \text { black to win } \$ 100 \\
25 \text { white to win } \$ 0 \\
50 \text { white to win } \$ 0\end{array}$ & $\begin{array}{l}25 \text { blue to win } \$ 50 \\
25 \text { blue to win } \$ 50 \\
50 \text { white to win } \$ 0\end{array}$ & 0.71 & 13.8 & 20.6 & 24.6 & 18.7 \\
\hline 9 & $\begin{array}{l}25 \text { black to win } \$ 100 \\
75 \text { white to win } \$ 0\end{array}$ & $\begin{array}{l}50 \text { blue to win } \$ 50 \\
50 \text { white to win } \$ 0\end{array}$ & 0.37 & 21.1 & 16.7 & 24.6 & 18.7 \\
\hline 13 & $\begin{array}{l}50 \text { white to lose } \$ 0 \\
25 \text { pink to lose } \$ 50 \\
25 \text { pink to lose } \$ 50\end{array}$ & $\begin{array}{l}50 \text { white to lose } \$ 0 \\
25 \text { white to lose } \$ 0 \\
25 \text { red to lose } \$ 100\end{array}$ & 0.65 & -20.6 & -13.8 & -20.4 & -24.8 \\
\hline 5 & $\begin{array}{l}50 \text { white to lose } \$ 0 \\
50 \text { pink to lose } \$ 50\end{array}$ & $\begin{array}{l}75 \text { white to lose } \$ 0 \\
25 \text { red to lose } \$ 100\end{array}$ & 0.31 & -16.7 & -21.1 & -20.4 & -24.8 \\
\hline 11 & $\begin{array}{l}25 \text { black to win } \$ 100 \\
25 \text { white to win } \$ 0 \\
50 \text { pink to lose } \$ 50\end{array}$ & $\begin{array}{l}50 \text { blue to win } \$ 50 \\
25 \text { white to lose } \$ 0 \\
25 \text { red to lose } \$ 100\end{array}$ & 0.24 & -15.5 & -34.5 & -8.8 & -15.3 \\
\hline
\end{tabular}

is not significant $(z=0.56)$. Therefore, in Choices 15, 13,19 , and 11, we cannot refute TAX with its previously estimated parameters and simplifying assumptions (Table 1).

Table 5 displays additional tests of coalescing. Choice 9 is the same as Choice 15, except for coalescing, as are Choices 13 and 5. CPT implies that there should be no differences due to splitting or coalescing. The TAX model, however (see Table 1), violates coalescing and predicts that people should prefer $G$ over $F$ in Choice 15, but should choose $F$ over $G$ in Choice 9. Table 5 shows that $71 \%$ of participants preferred $G$ in Choice 15, and only $37 \%$ chose $G$ in Choice 9, consistent with this prediction of the TAX model. Examining individual data, there were 76 par- ticipants who reversed preferences in this direction in the first replicate, compared to 16 who reversed preferences in the opposite direction $(z=6.26)$; in the second replicate the corresponding figures are 72 and 13 $(z=6.40)$. Examining these two choices on both replicates, the most frequent response pattern was to show this reversal on both replicates (38 people); only three people made the opposite reversal on both replicates $(z=5.47)$.

Similarly, significantly more people $(66 \%)$ chose $G$ in Choice 13, where two branches to lose $\$ 0$ were split, than did so in Choice $5(31 \%)$, where these two "good" branches were coalesced. Individual patterns confirm this reversal: 37 showed the same reversal of preferences on both replicates, compared to only five

Table 6 Tests of Risk Attitude, Loss Attitude, and Idempotence

\begin{tabular}{|c|c|c|c|c|c|c|c|}
\hline \multirow[b]{2}{*}{ No. } & \multicolumn{2}{|r|}{ Choice } & \multirow[b]{2}{*}{$\% S$} & \multicolumn{2}{|c|}{ Prior TAX } & \multicolumn{2}{|c|}{ Prior CPT } \\
\hline & $R$ & $S$ & & $R$ & $S$ & $R$ & $S$ \\
\hline 12 & $\begin{array}{l}50 \text { black to win } \$ 100 \\
50 \text { white to win } \$ 0\end{array}$ & $\begin{array}{l}50 \text { blue to win } \$ 50 \\
50 \text { green to win } \$ 50\end{array}$ & 0.67 & 33.3 & 50 & 37.4 & 50 \\
\hline 16 & $\begin{array}{l}50 \text { black to win } \$ 100 \\
50 \text { white to win } \$ 0\end{array}$ & $\begin{array}{l}100 \text { blue to win } \$ 50 \text { (win } \$ 50 \\
\text { for sure) }\end{array}$ & 0.69 & 33.3 & 50 & 37.4 & 50 \\
\hline 20 & $\begin{array}{l}50 \text { black to win } \$ 100 \\
50 \text { white to win } \$ 0\end{array}$ & $\begin{array}{l}100 \text { green to win } \$ 45 \text { (win } \$ 45 \\
\text { for sure) }\end{array}$ & 0.60 & 33.3 & 45 & 37.4 & 45 \\
\hline 6 & $\begin{array}{l}50 \text { white to lose } \$ 0 \\
50 \text { red to lose } \$ 100\end{array}$ & $\begin{array}{l}50 \text { pink to lose } \$ 50 \\
50 \text { orange to lose } \$ 50\end{array}$ & 0.37 & -33.3 & -50 & -40.8 & -50 \\
\hline 10 & $\begin{array}{l}50 \text { white to lose } \$ 0 \\
50 \text { red to lose } \$ 100\end{array}$ & $\begin{array}{l}100 \text { pink to lose } \$ 50 \text { (lose } \$ 50 \\
\text { for sure) }\end{array}$ & 0.31 & -33.3 & -50 & -40.8 & -50 \\
\hline 8 & $\begin{array}{l}50 \text { black to win } \$ 100 \\
50 \text { red to lose } \$ 100\end{array}$ & $\begin{array}{l}50 \text { white to win } \$ 0 \\
50 \text { white to lose } \$ 0\end{array}$ & 0.53 & -33.3 & 0 & -22.3 & 0 \\
\hline 18 & $\begin{array}{l}50 \text { black to win } \$ 100 \\
50 \text { red to lose } \$ 100\end{array}$ & $\begin{array}{l}100 \text { white marbles to win } \$ 0 \\
\text { (win } \$ 0 \text { for sure) }\end{array}$ & 0.38 & -33.3 & 0 & -22.3 & 0 \\
\hline $18 b$ & $\begin{array}{l}50 \text { black to win } \$ 100 \\
50 \text { red to lose } \$ 100\end{array}$ & $\begin{array}{l}100 \text { white marbles to win/lose } \\
\$ 0 \text { (no change for sure) }\end{array}$ & 0.52 & -33.3 & 0 & -22.3 & 0 \\
\hline 21 & $\begin{array}{l}50 \text { black to win } \$ 100 \\
50 \text { red to lose } \$ 100\end{array}$ & $\begin{array}{l}100 \text { yellow to lose } \$ 5 \text { (lose } \$ 5 \\
\text { for sure) }\end{array}$ & 0.48 & -33.3 & -5 & -22.3 & -5 \\
\hline
\end{tabular}


who showed the opposite reversal of preference on both replicates $(z=4.94)$. In the first and second replicates, 78 and 74 showed this reversal, compared to 17 and 14 who had the opposite reversals $(z=6.26$ and 6.40), respectively.

Choices 9, 5, and 11 provide a test of GLS in coalesced form (but with unequal numbers of branchestwo, two, and three). A very different picture emerges in this version of the test. Here, $63 \%$ preferred $F^{+}$ over $G^{+}$in Choice 9, 69\% preferred $F^{-}$over $G^{-}$in Choice 5 , and $76 \%$ preferred $F$ over $G$ in Choice 11; these modal choices are consistent with GLS. In sum, both the directions of preference among the subgambles and the conclusions regarding GLS are affected by whether gambles are presented in split or coalesced form. Note that all of the changing modal choices in Tables 4 and 5 are consistent with the predictions of the TAX model, with its previous parameters and its extending assumptions (Table 1).

Table 6 displays results for "filler" trials that assessed risk attitude, loss attitude, and idempotence. The parameterized models of TAX and CPT agree in their predictions in all cases in Table 6. Choices 12 and 16 differ only in that the two branches to win $\$ 50$ in Choice 12 have been coalesced to one branch in Choice 16, providing an indirect test of idempotence. CPT and TAX both satisfy idempotence and imply that there should be no difference between Choices 12 and 16 . The results indicate that $67 \%$ of participants preferred $S$ in Choice 12 and $69 \%$ preferred $S$ in Choice 16, consistent with idempotence. The finding that about two-thirds of the participants preferred the "safe" over the "risky" gamble with equal expected value indicates that these participants exhibited risk aversion, consistent with typical results. Choice 20 uses an even smaller value of sure cash and significantly more than half $(60 \%)$ still prefer the "safe" alternative of $\$ 45$, even though the expected value of the risky gamble is higher, $\$ 50$.

By idempotence, the preference should be the same in Choices 6 and 10, where the figures are 37\% and $31 \%$. Consistent with the reflection hypothesis (comparing 6 and 10 against 12 and 16), the majority are now risk seeking for these reflected gambles with nonpositive consequences; $63 \%$ and $69 \%$ preferring the gamble rather than accepting the sure loss of equal expected value.

Choices 8, 18, and 21 are tests of loss aversion. In Choice $8,53 \%$ showed loss aversion by preferring the certainty of neither winning nor losing to a 50-50 gamble to either win or lose $\$ 100$. Recall that these participants are playing with the endowment of $\$ 100$ "house" money, and perhaps that is why these participants are less loss averse than participants who make hypothetical choices involving their own money, as in, for example Tversky and Kahneman (1992), where participants were more strongly loss averse (see also Brooks and Zank 2005).

Choice 18 was designed as a test of idempotence, but during the experiment we realized that we had unintentionally introduced a type of consequenceframing effect. Our initial way to coalesce a 50-50 gamble to either win $\$ 0$ or lose $\$ 0$ was to describe it as a "sure thing to win $\$ 0 . "$ With this wording, $62 \%$ of 80 choices (by 40 people) chose the risky gamble over a sure thing to win $\$ 0$. However, with the more neutral wording (Choice 18b) of "no change for sure," only $52 \%$ preferred the sure thing $(n=138)$. When the sure thing was to lose $\$ 5$, participants were nearly indifferent between the gamble and the sure loss.

The data presented so far have been summarized by overall choice percentages. To examine whether these percentages are also characteristic of individuals, it is necessary to analyze detailed response patterns. The next section shows that such a detailed analysis confirms that violation of GLS in the overall choice percentages is also characteristic of individuals, but some violations not uncovered in the overall percentages were also detected.

\subsection{Error Analysis: Individual Data Patterns}

The property of GLS is directly analogous to transitivity, and the same statistical techniques can be used to test either property. Whereas transitivity can be written $A \succ B \cap B \succ C \Rightarrow A \succ C$, GLS can be written $G^{+} \succ F^{+} \cap G^{-} \succ F^{-} \Rightarrow G \succ F$. Both properties involve a three-choice relation, in which there are eight possible response patterns, assuming that ties are not allowed. In both cases, six of eight possible response combinations are compatible with the property, and two are not.

We use an error model to distinguish violations that occur by chance or error (response unreliability) from those that are intentional or true (consistent). In this analysis, it will be assumed that each person responds with his or her true preference with a fixed probability, and otherwise responds with an error. This analysis is similar to that used by Carbone and Hey (2000), Harless and Camerer (1994), Sopher and Gigliotti (1993), Birnbaum (2004b), and others, except that in the present treatment, error probabilities are estimated from preference reversals between replications of the same choices.

Because there are two repetitions of three choices, there are 64 possible response patterns for three choices in two replicates. Each participant falls into exactly one of these 64 cells. However, the frequencies in these cells are in most cases too small for analysis, so data are partitioned as follows. We tabulated the frequencies of the eight patterns that are repeated on both replicates, and the average frequencies for these eight patterns observed in either the 
first or second replicate, but not both. Thus, the model will fit 16 frequencies that are mutually exclusive and which sum to the total number of participants. From these 16 frequencies, there are three error probabilities and eight true probabilities (which sum to one) to be estimated.

Consider the probability that a participant will show an observed violation of GLS of the pattern $G^{+} G^{-} F$ in both replicates of the experiment. This can happen in eight different ways, corresponding to the eight possible true preference patterns. The conditional probability that a person would show the observed pattern of $G^{+} G^{-} F$ in both replicates given that the person's true pattern is $G^{+} G^{-} G$ (both replicates) is given by the following expression:

$$
\begin{aligned}
& P\left({ }^{\prime \prime} G^{+} G^{-} F, G^{+} G^{-} F^{\prime \prime} \mid G^{+} G^{-} G\right) \\
& \quad=\left(1-e_{1}\right)\left(1-e_{2}\right) e_{3}\left(1-e_{1}\right)\left(1-e_{2}\right) e_{3},
\end{aligned}
$$

where the quotation marks are used to distinguish an observed pattern of response (quotes) from a true pattern (without quotes); and $e_{1}, e_{2}$, and $e_{3}$ are the probabilities of error in the three choices, respectively. In this case, the participant expressed her or his preference correctly on two choices in each replicate and made an error on the third choice in both replicates. Therefore, the joint probability of having the true pattern is $G^{+} G^{-} G$ (on both replicates) and displaying this response pattern is given by

$$
\begin{aligned}
& P\left({ }^{\prime \prime} G^{+} G^{-} F, G^{+} G^{-} F^{\prime \prime} \cap G^{+} G^{-} G\right) \\
& \quad=a\left(G^{+} G^{-} G\right)\left(1-e_{1}\right)\left(1-e_{2}\right) e_{3}\left(1-e_{1}\right)\left(1-e_{2}\right) e_{3},
\end{aligned}
$$

where $a\left(G^{+} G^{-} G\right)$ is the theoretical true probability that a person's preference pattern is $G^{+} G^{-} G$.

The overall probability that a person exhibits this response pattern is the sum of eight terms like the above, representing each of the eight mutually exclusive, true patterns. Similarly, it is possible to write eight expressions for the average probability of showing each pattern on one replicate or the other, but not both.

The 16 frequencies sum to the number of participants, so there are 15 degrees of freedom in the data. The eight true probabilities must sum to one, so there are seven degrees of freedom in these eight probabilities; and three error probabilities, leaving $15-7-3=5$ degrees of freedom. The 16 observed frequencies are fit to this model by minimizing the $\chi^{2}(5)$ between observed and predicted frequencies.

Table 7 shows observed and fitted frequencies for the test of GLS in Choices 15, 13, and 11, in which all three choices involved three-branch gambles. The notation 000 in the table denotes the choices of $F^{+} \succ$ $G^{+}, F^{-} \succ G^{-}$, and $F \succ G$ in Choices 15, 13, and 11

\begin{tabular}{|c|c|c|c|c|c|c|c|}
\hline \multirow[b]{3}{*}{ Pattern } & \multicolumn{4}{|c|}{ Observed frequencies } & \multirow{2}{*}{\multicolumn{2}{|c|}{$\begin{array}{c}\text { Fitted } \\
\text { frequencies }\end{array}$}} & \multirow{3}{*}{$\begin{array}{l}\text { Estimated } \\
\text { true } \\
\text { probabilities }\end{array}$} \\
\hline & \multirow[b]{2}{*}{ Rep 1} & \multirow[b]{2}{*}{ Rep 2} & \multirow{2}{*}{$\begin{array}{l}\text { Both } \\
\text { reps }\end{array}$} & \multirow[b]{2}{*}{$U-I$} & & & \\
\hline & & & & & Both & $U-I$ & \\
\hline 000 & 16 & 17 & 5 & 11.5 & 5.9 & 11.3 & 0.08 \\
\hline 001 & 5 & 6 & 0 & 5.5 & 0.2 & 3.6 & 0 \\
\hline 010 & 24 & 29 & 12 & 14.5 & 11.1 & 15.0 & 0.18 \\
\hline 011 & 3 & 5 & 0 & 4 & 0.5 & 5.8 & 0 \\
\hline 100 & 36 & 30 & 10 & 23 & 9.8 & 21.5 & 0.10 \\
\hline 101 & 8 & 8 & 1 & 7 & 1.3 & 9.3 & 0 \\
\hline 110 & 63 & 54 & 29 & 29.5 & 29.6 & 29.2 & 0.51 \\
\hline 111 & 23 & 29 & 9 & 17 & 8.3 & 15.6 & 0.13 \\
\hline Total & 178 & 178 & 66 & 112 & 66.8 & 111.2 & 1 \\
\hline
\end{tabular}
(Table 3), respectively, where 0 denotes preference for
Table 7 Test of Gain-Loss Separability in Three-Branch Gambles (Choices 15, 13, and 11)

Notes. The estimated error rates are estimated to be $0.079,0.269$, and 0.166 for Choices 15,13, and 11, respectively; the estimated true probabilities of the sequences estimated from the true and error model are listed in the last column. The fit of the model had $\chi^{2}(5)=3.54$, ns. The entry set in bold is the pattern of violation of GLS predicted by the TAX model with its prior parameters. The column labeled " $\mathrm{U}$ - I" contains the average number for each pattern in two replicates minus the number who repeated that pattern (Union excluding the Intersection). Therefore, the sum of frequencies in "Both reps" and this column adds to the total number of participants $(66+112=178)$, and no response pattern is counted twice.

the $F$ gamble (or subgamble) and 1 denotes preference for the $G$ gamble (or subgamble). In this case, 110 represents the predicted pattern of violation by the TAX model, $G^{+} \succ F^{+} ; G^{-} \succ F^{-} ; F \succ G$. The estimated true probabilities of the eight patterns are listed in the rightmost column of Table 7, and the error probabilities are listed in the notes to that table.

According to the model, estimated error rates are $0.08,0.27$, and 0.17 for Choices 15, 13, and 11, respectively. The value of $\chi^{2}(5)=3.54$ is not significant, indicating an acceptable fit to the data. Note that the estimates in the model indicate that the most probable true pattern was the violation predicted by the TAX model (110); this pattern, $G^{+} G^{-} F$, had an estimated true probability of 0.51 . It was indeed the most commonly repeated pattern (29 participants showed this pattern in both replicates) as well as the most frequent pattern in both first and second replicates (63 and 54 , respectively).

When this true probability (of the violation pattern 110) was fixed to zero and all other parameters were free, the overall $\chi^{2}(6)$ increased to 25.74; therefore, $\chi^{2}(1)=25.74-3.54=22.2$, a significant difference. As a result, we can reject the hypothesis that the 110 pattern of violations can be attributed to error in favor of the hypothesis that people systematically violated GLS.

Other models with fewer parameters achieved reasonable approximations to the data. For example, the following special case achieved a fit of $\chi^{2}(11)=6.19$, an acceptable fit. The error probabilities were estimated to be (0), 0.36, and 0.18, respectively; and the 


\begin{tabular}{|c|c|c|c|c|c|c|c|}
\hline \multirow{3}{*}{ Table 8} & \multicolumn{7}{|c|}{$\begin{array}{l}\text { Test of Gain-Loss Separability in Two-, Two-, and } \\
\text { Three-Branch Gambles (Choices 9, 5, and 11) }\end{array}$} \\
\hline & \multicolumn{4}{|c|}{ Observed frequency } & \multirow{2}{*}{\multicolumn{2}{|c|}{$\begin{array}{l}\text { Fitted } \\
\text { frequency }\end{array}$}} & \multirow{3}{*}{$\begin{array}{c}\text { Estimated } \\
\text { true } \\
\text { probabilities }\end{array}$} \\
\hline & & & & & & & \\
\hline Pattern & Rep 1 & Rep 2 & reps & $U-I$ & Both & $U-I$ & \\
\hline 000 & 74 & 71 & 42 & 30.5 & 38.36 & 31.34 & 0.66 \\
\hline 001 & 13 & 17 & 5 & 10 & 5.09 & 11.63 & 0.08 \\
\hline 010 & 16 & 20 & 5 & 13 & 5.13 & 15.47 & 0.06 \\
\hline 011 & 5 & 8 & 2 & 4.5 & 1.64 & 6.46 & 0.01 \\
\hline 100 & 30 & 20 & 4 & 21 & 5.31 & 21.74 & 0 \\
\hline 101 & 9 & 13 & 1 & 10 & 1.41 & 6.97 & 0.01 \\
\hline 110 & 19 & 19 & 4 & 15 & 5.70 & 10.38 & 0.09 \\
\hline 111 & 12 & 10 & 5 & 6 & 5.07 & 6.31 & 0.09 \\
\hline Total & 178 & 178 & 68 & 110 & 67.70 & 110.30 & 1 \\
\hline
\end{tabular}

Notes. The estimated error rates are $0.27,0.14$, and 0.10 for Choices 9,5 , and 11 , respectively, and the estimated true probabilities of the sequences estimated from the model are listed in the last column. $\chi^{2}(5)=6.03$. The entry set in bold is the pattern of response predicted by the TAX model with its prior parameters.

probabilities of the eight true patterns were all fixed to zero except for $G^{+} G^{-} F, G^{+} G^{-} G$, and $F^{+} G^{-} F$, which had estimated true probabilities of $0.58,0.12$, and 0.30 , respectively. According to this model, the pattern of violation (110, i.e., $G^{+} G^{-} F$ ) was estimated to be the true pattern of $58 \%$ of the participants.

The true and error model was also fit to Choices 9, 5, and 11 (with two-, two-, and three-branch gambles), and results with all parameters free are shown in Table 8. Here, results are quite different, because the most frequently repeated pattern in this case satisfies GLS. This pattern (000), always choosing the $F$ gamble or subgamble, agrees with the prediction of the TAX model in this case. This pattern was estimated to be the "true" pattern of $66 \%$ of the participants. The error rates for the three choices were estimated to be $0.27,0.14$, and 0.10 , respectively. The overall $\chi^{2}(5)=6.03$ (not significant), with all parameters free. Setting the probabilities of both patterns of violation to zero (001 and 110), however, the $\chi^{2}(7)$ rose to 16.29 , for a difference of $\chi^{2}(2)=10.26$, both of which are significant. This test indicates that even though the modal choice probabilities in Table 1 satisfied GLS, this detailed analysis of response sequences in Table 8 indicates that small violations of GLS are significantly greater than zero.

The same model was fit to Choices 15, 13, and 19, which form a test of GLS with three-, three-, and four-branch gambles. Results are shown in Table 9. The modal pattern was now the 111 pattern $\left(G^{+} G^{-} G\right)$, which satisfies GLS. The $\chi^{2}(5)=2.89$, which is not significant. As a counterweight to the 111 pattern, the 010 and 100 patterns were now often repeated in both replicates, which contributed to the overall choice proportion between $G$ and $F$ being close to the TAX model prediction of 0.5 , even though the violation patterns 001 and 110 were rare in this case.
Table 9 Test of Gain-Loss Separability in Three-, Three-, and Four-Branch Gambles (Choices 15, 13, and 19)

\begin{tabular}{|c|c|c|c|c|c|c|c|}
\hline \multirow[b]{3}{*}{ Pattern } & \multicolumn{4}{|c|}{ Observed frequency } & \multirow{2}{*}{\multicolumn{2}{|c|}{$\begin{array}{c}\text { Fitted } \\
\text { frequency }\end{array}$}} & \multirow{3}{*}{$\begin{array}{l}\text { Estimated } \\
\text { true } \\
\text { probabilities }\end{array}$} \\
\hline & \multirow[b]{2}{*}{ Rep 1} & \multirow[b]{2}{*}{ Rep 2} & \multirow{2}{*}{$\begin{array}{l}\text { Both } \\
\text { reps }\end{array}$} & \multirow[b]{2}{*}{$U-I$} & & & \\
\hline & & & & & Both & $U-I$ & \\
\hline 000 & 15 & 16 & 3 & 12.5 & 4.46 & 9.30 & 0.06 \\
\hline 001 & 6 & 7 & 1 & 5.5 & 1.06 & 5.33 & 0.01 \\
\hline 010 & 18 & 25 & 10 & 11.5 & 10.44 & 11.73 & 0.19 \\
\hline 011 & 9 & 9 & 1 & 8 & 1.21 & 9.97 & 0 \\
\hline 100 & 23 & 21 & 9 & 13 & 9.33 & 13.11 & 0.17 \\
\hline 101 & 21 & 17 & 3 & 16 & 3.26 & 17.67 & 0 \\
\hline 110 & 24 & 30 & 4 & 23 & 4.37 & 19.91 & 0.02 \\
\hline 111 & 62 & 53 & 32 & 25.5 & 30.21 & 26.67 & 0.56 \\
\hline Total & 178 & 178 & 63 & 115 & 64.33 & 113.67 & \\
\hline
\end{tabular}

Notes. The estimated error rates are $0.078,0.221$, and 0.231 for Choices 15 , 13 , and 19 , respectively, and the estimated true probabilities of the sequences are listed in the last column. $\chi^{2}(5)=2.89$. The entry set in bold is the predicted pattern of TAX with the parameters and assumptions of Table 1.

In summary, depending on how branches are split or coalesced, the modal response pattern might be 110,000 , or 111 . The TAX model with previous parameters and simplifying assumptions (Table 1) predicted these modal patterns.

\section{Discussion}

The findings indicate that TAX is more accurate than CPT in predicting the data obtained in new tests of GLS and coalescing. With respect to the predictions in Table 3, we see that both OPT and CPT can be rejected by systematic violations of GLS.

The findings of this study are consistent with those of $\mathrm{Wu}$ and Markle (2004), who also concluded that GLS is violated, contradicting CPT. To account for the violations, however, they converted $\mathrm{CPT}$ into a configural CPT model (CCPT), where different weighting functions are used for gambles configured of nonpositive, nonnegative, and mixed consequences. Their model retains the use of cumulative weighting. Thus, whereas CCPT may be able to account for their data, it cannot account for our data, which show that tests of GLS depend on how branches are split or coalesced. Furthermore, CCPT cannot account for other "new paradoxes" that contradict CPT (Birnbaum 1999b, 2004a, b, 2005).

This study tested not only the behavioral properties that distinguish the models, but it also tested specific predictions implied by parameterized versions of the models. A test of indifference was constructed from the TAX model and the simplifying assumptions of Table 1, which included the assumption that $u(x)=x$ for both positive and negative consequences near zero. Despite high power in the test (proportions outside the range 0.45 to 0.55 would have been 
rejected), the observed proportion at this point prediction was 0.517 , acceptably close to 0.500 , based on the prediction of indifference.

This TAX model can account for a variety of phenomena in risky decision making, including the original and extended forms of Allais common consequence paradoxes, common ratio paradox, violations of restricted branch independence, violations of lower and upper cumulative independence, violations of upper-tail independence, violations of coalescing, and violations of first-order stochastic dominance (Birnbaum 1999a, b, 2004a, b, 2005). These findings have been called new paradoxes because they violate CPT in the same sense that the Allais paradoxes violate EU. Furthermore, TAX fits majority choices in studies of different phenomena, obtained under uniform conditions, using the same parameters.

It is worth repeating that this simple TAX model implies risk aversion, loss aversion, and reflection, as well as violations of coalescing and GLS without using any assumption that utilities of losses loom larger than gains, as is done in CPT. In this TAX model, loss aversion in mixed gambles is described by greater weight assigned negative consequences rather than by amplification of negative utilities. It might seem arbitrary to attribute loss aversion to weight rather than utility; however, it is this distinction that allows TAX to predict violation of GLS.

Both TAX and CPT account for tests of risk aversion and loss aversion in Table 6. Consistent with previous findings (e.g., Tversky and Kahneman 1992), we found that the majority of participants preferred "safe gambles" with nonnegative consequences over risky ones in 50-50 gambles (e.g., Choice 12). Similarly, with strictly nonpositive consequences, the majority exhibit risk-seeking behavior (e.g., Choice 6), also consistent with previous findings. With mixed gambles, people slightly favor the safe gamble over the risky one (e.g., Choice 8), showing evidence of loss aversion.

In this study, where participants were endowed with $\$ 100$ and thus risked only "house" money, participants were not as loss averse as has been observed in studies where participants make hypothetical choices involving possible losses of their own money (cf. Battalio et al. 1990). It would be interesting to determine whether our results would be much impacted by the use of hypothetical rather than potentially real losses. One of the practical problems in this line of research is how to implement real losses without endowing participants or perhaps violating ethical rules for conduct of behavioral research by taking money from our participants. See Brooks and Zank (2005) for further discussion of this issue.

As noted by Wu and Markle (2004), GLS is assumed in most treatments of loss aversion, the tendency for people to avoid 50-50 bets to win or lose equal amounts (Abdellaoui et al. 2005, Fennema and van Assen 1998, Köbberling and Wakker 2005, Schmidt and Traub 2002). They note that theories and indices of loss aversion based on the assumption of GLS will be incorrect to the extent that GLS is not descriptive. Brooks and Zank (2005) report that loss aversion depends on the sign of the common consequence on a comonotonic branch. If this reported violation of comonotonic independence holds up, it provides an additional piece to the empirical puzzle, and further illustrates the risks of assuming theoretical models in our definitions of empirical phenomena (Schmidt and Zank 2005).

It would be interesting to know if there are situations where TAX would be forced to allow the utility function to be kinked at zero. There is nothing in the TAX model that rules out such a kink; the present data simply do not require it, once the TAX model is used. We doubt that the utility function used in this model, $u(x)=x$, will extrapolate to consequences with large absolute values. The model as stated, with $u(x)=x$ on an unrestricted range, implies that multiplication of all consequences by a constant in a choice should not reverse that choice. The TAX model required a nonlinear function $u(x)=x^{0.8}$ to perfectly fit the Kahneman and Tversky (1979) data, which involved hypothetical wins or losses comparable to a month's wages. However, are there data that also require $u(x) \neq-u(-x)$ for the TAX model?

Similarly, we suspect that our use of a single value of the configural weight parameter for both positive and mixed gambles is an oversimplification. That assumption, combined with $u(x)=x$, implies that addition of a constant to all consequences should not reverse a choice. However, we suspect that the degree of risk aversion in gambles on small positive consequences may be stronger than the comparable degree of loss aversion in the same gambles translated to mixed gambles. In addition to these testable implications, the TAX model (Table 1) contains discontinuities and violations of consequence monotonicity (Birnbaum 1997) that would be worthwhile to investigate both theoretically and empirically.

It is not only this version of TAX that makes discontinuous predictions. Edward's (1962) suggestion that there are different pages in a book of weights for different gambles with positive, negative, and mixed consequences as well as the revised CCPT model of $\mathrm{Wu}$ and Markle (2004) also imply discontinuities as a consequence is increased, converting a purely negative gamble, for example, into a mixed gamble. Any such model in which parameters suddenly change as one "turns the page" from pure gains gambles to gambles containing gains and the consequence zero, for example, can produce such effects. Indeed, violations of consequence monotonicity have 
been observed in judgments buying and selling prices of gambles as small positive or negative consequences 三 ire changed to zero (Birnbaum 1997).

A rival to the "book of weights" approach would be to model configural weights with continuous functions that do not exhibit discontinuities from page to page. For example, in the TAX model, the configural transfer parameter might be modeled as a smooth function of the utility of the gamble. This way, as one changes from gambles with positive to negative utilities, the transfer parameter might decrease to zero and reverse sign, rather than show a discontinuous jump, as in Table 1.

Our tests allow that idempotence can be retained, pending further tests. Such tests, of course, do not show that the property will always be satisfied. Our tests were not designed to investigate specific implications of any particular model that violates idempotence, which would be a preferable way to test such a property. Meginniss (1976) proposed such a model, hich violates idempotence. Marley and Luce (2005) plore such models from a theoretical perspective; but these models have not yet been fit to data to provide specific predictions of violation. It would be useful to devise a theorem that if certain violations of coalescing are observed, for example, then a particular violation of idempotence should be observed. Such a theorem would then be tested as a behavioral property to assess such models.

Although this study did not directly test the property of duplex decomposition (Luce 2000, p. 213), there are some possible connections between GLS and duplex decomposition that deserve mention. Duplex decomposition can be written as follows:

$$
G \sim G^{-} \oplus G^{+}
$$

where $G$ is a mixed gamble whose positive and negative subgambles are $G^{+}$and $G^{-}$, and $\oplus$ represents the joint receipt of two independent gambles. Under the assumption that $U$ is additive over $\oplus$, and with general segregation and other assumptions (Luce 2000, p. 215), the same rank and sign-dependent representation (Luce and Fishburn 1991, 1995) as used in CPT follows, which satisfies GLS. Therefore, failure of GLS shows that not all of the assumptions leading to that representation can be correct.

A second approach is described in Luce (2000, Chapter 7), where it is assumed that it is value rather than utility that is additive over joint receipts, and utility is polynomial additive over gains and losses separately. Here, different assumptions concerning duplex decomposition and the utility functions lead to different representations for the mixed case, which Luce (2000, pp. 243-250) worked out for binary gambles. These representations differ from that in CPT.
Whereas this study made use of three- and fourbranch gambles to produce violations of GLS, Wu and Markle (2004) reported violations of GLS in binary gambles as well. Assuming that their results can be replicated, it would be worthwhile to apply the error model of our paper to test empirically whether joint receipts would also show the same violations of GLS. It seems likely that if binary gambles violate GLS, joint receipts might also violate GLS in the same way (which they would if $G \sim G^{-} \oplus G^{+}$), but these are clearly empirical questions. That is, one can test whether $G^{+} \oplus G^{-} \prec F^{+} \oplus F^{-}$in cases where $G \prec F$, and yet $G^{+} \succ F^{+} \cap G^{-} \succ F^{-}$. This property would be worthwhile to test in conjunction with GLS in binary gambles because its results would strongly restrict the possible representations of mixed gambles.

In conclusion, we found that directions of preference and conclusions regarding GLS are affected by whether gambles are presented in split or coalesced form. Our results show that we might have concluded that GLS was violated, weakly violated, or satisfied depending on exactly how the GLS test was constructed. These results rule out theories such as CPT that imply coalescing and GLS; they also rule out models such as CCPT, which satisfy coalescing.

An online supplement to this paper is available on the Management Science website at http://mansci. pubs.informs.org/ecompanion.html.

\section{Acknowledgments}

The authors thank two anonymous reviewers for helpful suggestions that improved the exposition. Support was received from National Science Foundation grants SBR9410572, SES 99-86436, and BCS-0129453.

\section{References}

Abdellaoui, M., H. Bleichrodt, C. Paraschiv. 2005. Measuring loss aversion: A parameter-free approach. Working paper, Erasmus University and GRID, ENSAM-P. aris and ESTP, France.

Battalio, R., J. H. Kagel, K. Jiranyakul. 1990. Testing between alternative models of choice under uncertainty: Some initial results. J. Risk Uncertainty 3 25-30.

Birnbaum, M. H. 1974. The nonadditivity of personality impressions. J. Experiment. Psych. 102 543-561.

Birnbaum, M. H. 1999a. Paradoxes of Allais, stochastic dominance, and decision weights. J. Shanteau, B. A. Mellers, D. A. Schum, eds. Decision Science and Technology: Reflections on the Contributions of Ward Edwards. Kluwer Academic Publishers, Norwell, MA, 27-52.

Birnbaum, M. H. 1999b. Testing critical properties of decision making on the Internet. Psych. Sci. 10 399-407.

Birnbaum, M. H. 2004a. Causes of Allais common consequence paradoxes: An experimental dissection. J. Math. Psych. 48(2) 87-106.

Birnbaum, M. H. 2004b. Tests of rank-dependent utility and cumulative prospect theory in gambles represented by natural frequencies: Effects of format, event framing, and branch splitting. Organ. Behav. Human Decision Processes 95 40-65.

Birnbaum, M. H. 2005. Three new tests of independence that differentiate models of risky decision making. Management Sci. 51 1346-1358. 
Birnbaum, M. H., A. Chavez. 1997. Tests of theories of decision making: Violations of branch independence and distribution independence. Organ. Behav. Human Decision Processes 71(2) 161-194.

Birnbaum, M. H., S. E. Stegner. 1979. Source credibility in social judgment: Bias, expertise, and the judge's point of view. J. Personality Soc. Psych. 37 48-74.

Brooks, P., H. Zank. 2005. Loss averse behavior. J. Risk Uncertainty 31 301-325.

Fennema, H., M. van Assen. 1998. Measuring the utility of losses by means of the tradeoff method. J. Risk Uncertainty 17 277-295.

Humphrey, S. J. 1995. Regret aversion or event-splitting effects? More evidence under risk and uncertainty. J. Risk Uncertainty 11 263-274.

Kahneman, D., A. Tversky. 1979. Prospect theory: An analysis of decision under risk. Econometrica 47 263-291.

Koebberling, V., P. P. Wakker. 2005. An index of loss aversion. J. Econom. Theory 122 119-131.

Levy, H., M. Levy. 2002. Prospect theory, much ado about nothing? Management Sci. 48 1334-1349.

Luce, R. D. 2000. Utility of Gains and Losses: Measurement-Theoretical and Experimental Approaches. Lawrence Erlbaum Associates, Mahwah, NJ.

Luce, R. D., P. C. Fishburn. 1991. Rank and sign-dependent linear utility models for finite first order gambles. J. Risk Uncertainty 4 29-59.

Luce, R. D., P. C. Fishburn. 1995. A note on deriving rank-dependent utility using additive joint receipts. J. Risk Uncertainty 11 5-16.

Luce, R. D., A. A. Marley. 2000. On elements of chance. Theory Decision 49(2) 97-126.
Marley, A. A. J., R. D. Luce. 2005. Independence properties vis-à-vis several utility representations. Theory Decision 58 77-143.

Quiggin, J. 1982. A theory of anticipated utility. J. Econom. Behav. Organ. 3 324-345.

Quiggin, J. 1993. Generalized Expected Utility Theory: The RankDependent Model. Kluwer, Boston, MA.

Schmidt, U., S. Traub. 2002. An experimental test of loss aversion. J. Risk Uncertainty 25 233-249.

Schmidt, U., H. Zank. 2005. What is loss aversion? J. Risk Uncertainty 30 157-167.

Sopher, B., G. Gigliotti. 1993. Intransitive cycles: Rational choice or random error? An answer based on estimation of error rates with experimental data. Theory Decision 35 311-336.

Starmer, C. 2000. Developments in non-expected utility theory: The hunt for a descriptive theory of choice under risk. J. Econom. Literature 38 332-382.

Starmer, C., R. Sugden. 1993. Testing for juxtaposition and eventsplitting effects. J. Risk Uncertainty 6 235-254.

Tversky, A., D. Kahneman. 1992. Advances in prospect theory: Cumulative representation of uncertainty. J. Risk Uncertainty 5 297-323.

Wakker, P. 2003. The data of Levy and Levy (2002), "Prospect theory: Much ado about nothing?" Support prospect theory. Management Sci. 49 979-981.

Wu, G., A. B. Markle. 2004. An empirical test of gain-loss separability in prospect theory. Working Manuscript 06-25-04, Graduate School of Business, University of Chicago, http://gsbwww. uchicago.edu/fac/george.wu/research/abstracts.html. 


\section{Comments on mnsc0592}

\section{Page: 1}

Sequence number: 1

Author: Production Editor

Date: 9/26/2006 11:12:52 AM

Type: Note

Please provide/confirm author addresses and e-mails.

\section{Page: 2}

Sequence number: 1

Author: Production Editor

Date: 9/26/2006 11:18:04 AM

Type: Highlight

GPU

Sequence number: 2

Author: Production Editor

Date: 9/26/2006 11:18:21 AM

Type: Note

Is this correct?

\section{Page: 3}

Sequence number: 1

Author: Production Editor

Date: 9/26/2006 11:23:41 AM

Type: Highlight

Edwards (1962),

Sequence number: 2

Author: Production Editor

Date: 9/26/2006 11:23:59 AM

Type: Note

Please insert cite into references.

Sequence number: 3

Author: Production Editor

Date: 9/26/2006 11:24:37 AM

Type: Note

OK to delete quotation marks?

Sequence number: 4

Author: Production Editor

Date: 9/26/2006 11:25:03 AM

Type: Highlight

Birnbaum

Sequence number: 5

Author: Production Editor

Date: 9/26/2006 11:25:06 AM

Type: Highlight

and Navarrete 1998,

Sequence number: 6

Author: Production Editor 
Date: 9/26/2006 11:25:19 AM

Type: Note

Please insert cite into references.

Sequence number: 7

Author: Production Editor

Date: 9/26/2006 11:26:30 AM

Type: Highlight

Luce and Marley (2005)

Sequence number: 8

Author: Production Editor

Date: 9/26/2006 11:27:47 AM

Type: Note

Is this correct (as in references) or should it be 'Marley and Luce 2005' an additional reference (which would need to be inserted in reference section)?

Sequence number: 9

Author: Production Editor

Date: 9/26/2006 11:28:14 AM

Type: Highlight

expected utility

Sequence number: 10

Author: Production Editor

Date: 9/26/2006 11:28:18 AM

Type: Highlight

EU).

Sequence number: 11

Author: Production Editor

Date: 9/26/2006 11:28:21 AM

Type: Highlight

as in EU).

Sequence number: 12

Author: Production Editor

Date: 9/26/2006 11:28:31 AM

Type: Note

Edits correct?

\section{Page: 4}

Sequence number: 1

Author: Production Editor

Date: 9/26/2006 12:11:38 PM

Type: Highlight

p07,

Sequence number: 2

Author: Production Editor

Date: 9/26/2006 12:11:47 PM

Type: Note

Edit OK?

Sequence number: 3

Author: Production Editor

Date: 9/26/2006 12:12:54 PM

Type: Highlight

expected value

Sequence number: 4

Author: Production Editor

Date: 9/26/2006 12:13:03 PM

Type: Note 


\section{Page: 5}

Sequence number: 1

Author: Production Editor

Date: 9/26/2006 12:14:32 PM

Type: Note

Edit correct?

\section{Page: 6}

Sequence number: 1

Author: Production Editor

Date: 9/26/2006 12:15:09 PM

Type: Note

Edit correct?

\section{Page: 8}

Sequence number: 1

Author: Production Editor

Date: 9/26/2006 12:16:39 PM

Type: Note

OK to delete quotation marks?

Sequence number: 2

Author: Production Editor

Date: 9/26/2006 12:25:52 PM

Type: Highlight

Carbone

Sequence number: 3

Author: Production Editor

Date: 9/26/2006 12:25:54 PM

Type: Highlight

and Hey (2000),

Sequence number: 4

Author: Production Editor

Date: 9/26/2006 12:26:13 PM

Type: Note

Insert cite into references.

Sequence number: 5

Author: Production Editor

Date: 9/26/2006 12:26:25 PM

Type: Highlight

Harless and Camerer (1994),

Sequence number: 6

Author: Production Editor

Date: 9/26/2006 12:26:51 PM

Type: Note

Insert cite into references. 


\section{Page: 11}

Sequence number: 1

Author: Production Editor

Date: 9/26/2006 12:31:07 PM

Type: Highlight

Köbberling

Sequence number: 2

Author: Production Editor

Date: 9/26/2006 12:31:22 PM

Type: Note

Confirm correct spelling for author.

Sequence number: 3

Author: Production Editor

Date: 9/26/2006 12:32:29 PM

Type: Highlight

Birnbaum 1997)

Sequence number: 4

Author: Production Editor

Date: 9/26/2006 12:33:05 PM

Type: Note

Insert cite into references.

Sequence number: 5

Author: Production Editor

Date: 9/26/2006 12:33:10 PM

Type: Highlight

Edward's

Sequence number: 6

Author: Production Editor

Date: 9/26/2006 12:33:36 PM

Type: Note

Insert cite into references.

\section{Page: 12}

Sequence number: 1

Author: Production Editor

Date: 9/26/2006 12:33:50 PM

Type: Highlight

Birnbaum 1997).

Sequence number: 2

Author: Production Editor

Date: 9/26/2006 12:34:04 PM

Type: Note

Insert cite into references.

Sequence number: 3

Author: Production Editor

Date: 9/26/2006 12:34:32 PM

Type: Highlight

Meginniss (1976)

Sequence number: 4

Author: Production Editor

Date: 9/26/2006 12:36:00 PM

Type: Note

Insert cite into references. 
Sequence number: 5

Author: Production Editor

Date: 9/26/2006 12:36:11 PM

Type: Highlight

Marley and Luce (2005)

Sequence number: 6

Author: Production Editor

Date: 9/26/2006 12:36:22 PM

Type: Note

Edit correct?

Sequence number: 7

Author: Production Editor

Date: 9/26/2006 12:36:51 PM

Type: Highlight

Luce (2000,

Sequence number: 8

Author: Production Editor

Date: 9/26/2006 12:36:54 PM

Type: Highlight

Chapter 7),

Sequence number: 9

Author: Production Editor

Date: 9/26/2006 12:37:19 PM

Type: Note

Also insert pages.

Sequence number: 10

Author: Production Editor

Date: 9/26/2006 12:38:48 PM

Type: Highlight

GRID,

Sequence number: 11

Author: Production Editor

Date: 9/26/2006 12:38:49 PM

Type: Highlight

ENSAM-

Sequence number: 12

Author: Production Editor

Date: 9/26/2006 12:38:51 PM

Type: Highlight

ESTP,

Sequence number: 13

Author: Production Editor

Date: 9/26/2006 12:39:33 PM

Type: Note

Should abbreviations be spelled out?

\section{Page: 13}

Sequence number: 1

Author: Production Editor

Date: 9/26/2006 12:40:30 PM

Type: Highlight

Koebberling,

Sequence number: 2

Author: Production Editor

Date: 9/26/2006 12:40:44 PM 
Type: Note

Confirm correct spelling here and throughout.

Sequence number: 3

Author: Production Editor

Date: 9/26/2006 12:41:29 PM

Type: Note

Please review text: is this correct author order?

Sequence number: 4

Author: Production Editor

Date: 9/26/2006 12:43:07 PM

Type: Note

Cited as 'Luce and Marley' in text. Author order correct? 